\title{
Changes in Anthocyanin and Antioxidant Contents during Maturation of Australian Highbush Blueberry (Vaccinium corymbosum L.) Cultivars ${ }^{\dagger}$
}

\author{
Joel B. Johnson ${ }^{1, *}{ }^{\mathbb{D}}$, Michelle Steicke ${ }^{2}$, Janice S. Mani ${ }^{1}$, Shiwangni Rao ${ }^{2}$, Scott Anderson ${ }^{3}{ }^{(}$, Lara Wakeling $^{2}$ \\ and Mani Naiker ${ }^{1}$ \\ 1 School of Health, Medical \& Applied Sciences, Central Queensland University, Rockhampton, \\ QLD 4701, Australia; janice.mani@gmail.com (J.S.M.); m.naiker@cqu.edu.au (M.N.) \\ 2 School of Health and Life Sciences, Federation University Australia, Ballarat, VIC 3350, Australia; \\ michellesteicke@hotmail.com (M.S.); wani.rao@gmail.com (S.R.); 1.wakeling@federation.edu.au (L.W.) \\ 3 Faculty of Health, Deakin University, Waurn Ponds, Geelong, VIC 3216, Australia; scott@sda0.com \\ * Correspondence: joel.johnson@cqumail.com \\ + Presented at the 2nd International Electronic Conference on Applied Sciences, 15-31 October 2021; Available \\ online: https:/ / asec2021.sciforum.net/.
}

check for updates

Citation: Johnson, J.B.; Steicke, M.; Mani, J.S.; Rao, S.; Anderson, S.; Wakeling, L.; Naiker, M. Changes in Anthocyanin and Antioxidant Contents during Maturation of Australian Highbush Blueberry (Vaccinium corymbosum L.) Cultivars. Eng. Proc. 2021, 11, 6. https:// doi.org/10.3390/ASEC2021-11155

Academic Editor: Nunzio Cennamo

Published: 15 October 2021

Publisher's Note: MDPI stays neutral with regard to jurisdictional claims in published maps and institutional affiliations.

Copyright: (c) 2021 by the authors. Licensee MDPI, Basel, Switzerland. This article is an open access article distributed under the terms and conditions of the Creative Commons Attribution (CC BY) license (https:// creativecommons.org/licenses/by/ $4.0 /)$.

\begin{abstract}
The Australian blueberry industry is worth over $\$ 300$ million, but there is limited information on factors influencing their chemical composition, particularly their ripeness and harvest stage. This pilot study investigated changes in total monomeric anthocyanin content (TMAC; measured using the $\mathrm{pH}$-differential method) and total antioxidant capacity (TAC; measured with the cupric reducing antioxidant capacity assay) of four Australian highbush blueberry cultivars (Denise, Blue Rose, Brigitta and Bluecrop) at four time points and three maturity stages (unripe, moderately ripe and fully ripe). The TAC of most cultivars decreased by $8-18 \%$ during ripening, although that of the Blue Rose cultivar increased markedly. However, the TAC of ripe fruit from this cultivar also fluctuated markedly throughout the harvest season (between 1168-2171 mg Trolox equivalents $100 \mathrm{~g}^{-1}$ ). The TMAC increased sharply between the medium-ripe and fully ripe maturity stages, with the Blue Rose cultivar showing the highest TMAC values $\left(211 \mathrm{mg} 100 \mathrm{~g}^{-1}\right.$, compared to 107-143 mg $100 \mathrm{~g}^{-1}$ for the remaining varieties). The TMAC of ripe fruit from this cultivar also rose steadily throughout the harvest season, in contrast to most other cultivars where the TMAC fell slightly over time. These results indicate that the levels of health-benefitting compounds in Australian-grown highbush blueberries may depend not only on the cultivar, but also upon the time of harvest.
\end{abstract}

Keywords: ripening; phytochemical composition; functional food; blueberry

\section{Introduction}

Highbush blueberries (Vaccinium corymbosum L.) are the second-most grown berry crop in Australia, second to strawberries. After being commercially established in Victoria in 1974, rapid growth in the past 15 years has seen a 10-fold expansion in the blueberry industry value to reach $\$ 300$ million farmgate value in 2019 [1]. Most of the crop (75\%) is consumed fresh by the domestic market, with 15\% used in domestic processing [1].

Blueberries are a well-known functional food, with purported health benefits including antioxidative, anti-inflammatory, neuroprotective, anti-obesity, anti-diabetic and cardioprotective effects [2]. The majority of these health benefits are derived from their high levels of anthocyanins and polyphenols [3]. At least 25 different anthocyanins have been identified in highbush blueberries, with malvidin, delphinidin and peonidin being the predominant aglycones (anthocyanidins) present $[4,5]$. The phenolic acids present are similarly diverse, with hydroxycinnamic acid esters (in particular chlorogenic acid) found to be the most abundant polyphenols [6]. Both the anthocyanins and polyphenols present in blueberries contribute to the exceptional antioxidant capacity of these matrices. 
Previous studies have investigated changes in anthocyanin and phenolic content throughout the ripening process in highbush blueberries [7-9], generally finding a marked increase in anthocyanin content during maturation, accompanied by decreasing total phenolic content and antioxidant capacity. Both genotype and environment influence the accumulation process and final content of anthocyanins and phenolic compounds in blueberries $[4,9,10]$. However, there is limited information available on the effect of growing conditions and other physiological factors on anthocyanin content [11].

Furthermore, there is little published literature available on the phytochemical composition of Australian-grown blueberries, with previous studies comparing the anthocyanin content of ripe fruit between different cultivars [5] or studying the effects of varying food preservation techniques on anthocyanin content [12]. Furthermore, there does not appear to be any previous work investigating the changes in anthocyanin and antioxidant capacity during the ripening process of Australian-grown blueberry cultivars. Consequently, the aim of this study was to undertake a one-year pilot study to investigate the changes in anthocyanin content and antioxidant capacity in Australian highbush blueberries throughout different stages of berry development.

\section{Methods}

\subsection{Blueberry Sample Preparation}

Four northern highbush blueberry cultivars were included in this study (Denise, Blue Rose, Brigitta and Bluecrop). Brigitta was originally developed in Australia and has now become popular worldwide due to its excellent storage and shipping characteristics. Blueberry samples were collected from a farm in Buninyong, western Victoria (Buninyong Blueberry Farm) during the 2015 summer harvest season. The sampling time points were at approximately weekly intervals for four weeks, on the 16, 23 and 30 January, and 12 February. At each sampling time point, ripe, medium-ripe and unripe blueberries were collected (where available for each variety), based on the appearance, colour and hardness of the fruit. Ripeness was qualitatively determined, with dark purple berries classified as ripe, reddish berries classified as medium-ripe, and green berries classified as unripe. For each sample, approximately $200 \mathrm{~g}$ of berries were collected across the rows for each cultivar, ensuring that all positions on the plants were sampled. The samples were stored at $-20{ }^{\circ} \mathrm{C}$ prior to extraction.

\subsection{Extraction of Anthocyanins and Phenolics}

For each sample, approximately $20 \mathrm{~g}$ of frozen berries were subsampled and homogenised in a mortar and pestle. Extractions were performed in triplicate, using around $5 \mathrm{~g}$ of the homogenate in $15 \mathrm{~mL}$ of extraction solvent (95\% methanol; $5 \%$ glacial acetic acid). The extracts were shaken at $250 \mathrm{rpm}$ for $10 \mathrm{~min}$ (Ratek orbital shaker), followed by centrifugation $(10,000 \mathrm{rpm} ; 10 \mathrm{~min})$ and collection of the supernatant. The extraction was repeated twice more on the sample pellet, with the combined supernatant made up to $50 \mathrm{~mL}$, vacuum filtered (Whatman No. 1) and stored at $-20{ }^{\circ} \mathrm{C}$.

\subsection{Measurement of Total Anthocyanin Content and Antioxidant Capacity}

Total monomeric anthocyanin content (TMAC) was measured on the triplicate extracts using the pH-differential method, as previously described [13]. Results were expressed as equivalents of cyanidin-3-glucoside. The total antioxidant capacity (TAC) of the extracts was determined on the triplicate extracts using the previously described CUPRAC proto$\mathrm{col}$ [13]. From the absorbance at $450 \mathrm{~nm}$, TAC results were quantified as a function of the equivalent absorbance of Trolox standards $\left(R^{2}=0.99\right)$.

\section{Results and Discussion}

\subsection{Changes in Anthocyanin Content and Antioxidant Capacity during Maturation}

The first aim of this study was to determine the changes in anthocyanin content and antioxidant capacity at different ripeness stages. In order to do this, ripe, medium- 
ripe and unripe blueberry samples for each cultivar were harvested on the same date (16 January 2015) and subsequently analysed.

The range of TAC and TMAC values found across all cultivars and maturity stages generally agreed with the range of results reported by Connor et al. [10] in 16 varieties of highbush blueberries grown in the United States. As shown in Figure 1a, the total antioxidant capacity of the blueberry samples generally decreased throughout the ripening process, as previously reported by several authors [7-9]. However, not every cultivar followed this trend, with the TAC of the Brigitta cultivar increasing between the mediumripe and ripe stages (Figure 1a). The TAC of the Blue Rose cultivar showed the greatest deviation, increasing markedly between the unripe and ripe stages. However, as no medium-ripe fruit could be obtained for this variety, further investigation is required to confirm this trend.
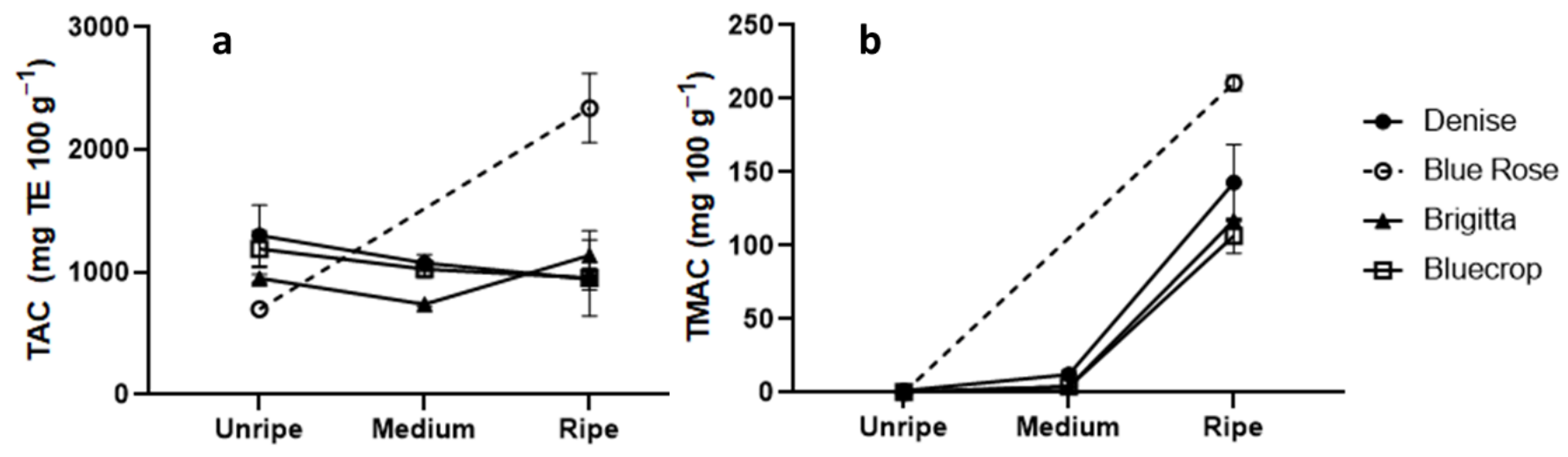

Figure 1. (a) There was no clear trend visible in the total antioxidant capacity (TAC) of the four blueberry varieties at different stages of ripeness (unripe, medium-ripe and ripe). All samples were collected on the same date (16 January 2015) to avoid potential effects of temporal variation. Note that no sample of "medium" ripeness was available for the Blue Rose cultivar. (b) Total monomeric anthocyanin content (TMAC) increased markedly in the four blueberry cultivars at different stages of ripeness. All samples were collected on the same date (16 January 2015). Note that no sample of "medium" ripeness was available for the Blue Rose cultivar.

The total anthocyanin content increased in a non-linear fashion throughout the maturation process, with a sharp increase between the medium and ripe stages (Figure 1b), as previously documented in other cultivars [7,9]. However, some previous researchers only recorded the development of anthocyanin content in already ripened fruit [8], rather than the changes from unripe to ripe fruits, as presented here. This development of anthocyanin content during the ripening process occurs as a temporally-dependent extension of the flavonoid synthesis pathway, primarily controlled by the transcription factor MYB1 [14].

As observed with the TAC, the final TMAC found in ripe fruits from the Blue Rose

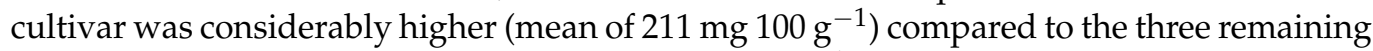
cultivars (means ranging between 107-143 mg $100 \mathrm{~g}^{-1}$ ), highlighting the opportunity for further investigation of the phytochemical constituents and potential health benefits of this specific cultivar. Overall, the anthocyanin content of all cultivars fell within the average range reported by Stevenson and Scalzo [4] for 80 different blueberry genotypes.

\subsection{Anthocyanin Content and Antioxidant Capacity in Ripe Fruit at Different Timepoints during the Season}

The second aim of this study was to investigate if there is temporal variation in the anthocyanin content and antioxidant capacity of blueberry fruit at different times within the harvest season. In order to investigate this possibility, ripe fruit from each cultivar were collected at four different sampling timepoints (mid-Jan to mid-Feb) and analysed.

Neither the TAC or TMAC showed any clear inter-varietal trends throughout the harvest season (Figure 2); however, there were significant changes associated with specific 
varieties. Both Denise and Bluecrop showed a slight reduction in TAC throughout the season, while the TAC of Blue Rose fell sharply in late January before increasing again. The TAC of Brigitta increased around the end of January, before falling back to its original levels by mid-February.
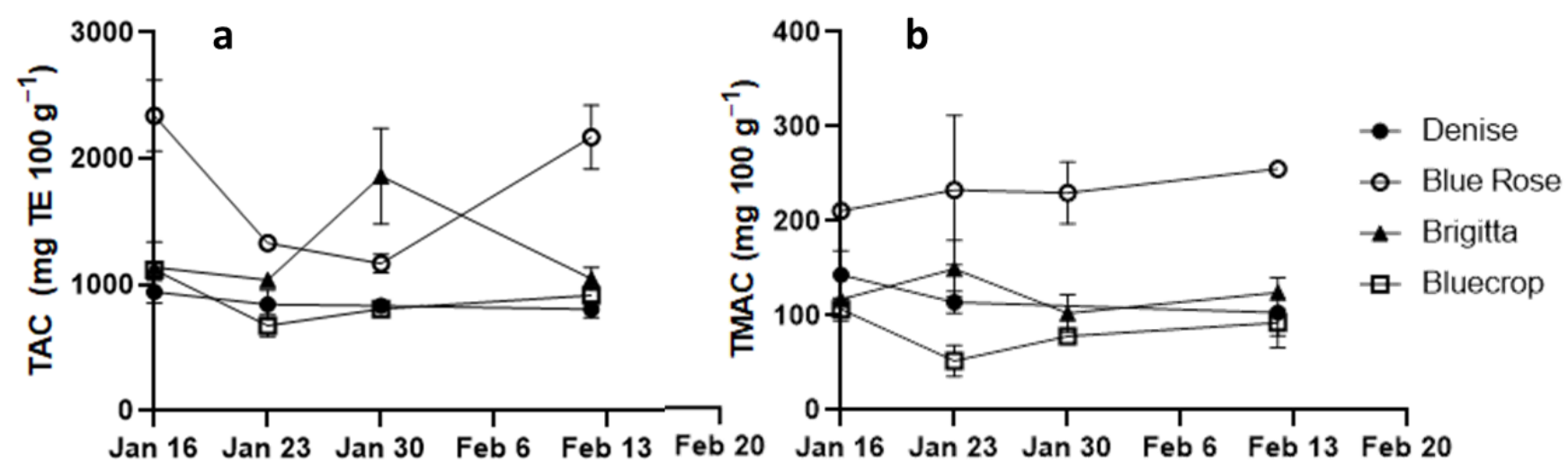

Figure 2. (a) Variation in the total antioxidant capacity (TAC) of ripe blueberries from four different cultivars throughout the harvest season. (b) Variation in the total monomeric anthocyanin content (TMAC) of ripe blueberries from four different cultivars throughout the harvest season.

The anthocyanin content of the Blue Rose cultivar increased steadily throughout the harvest season (Figure 2b), while that of Denise showed a slight fall. The anthocyanin content of Brigitta and Bluecrop fluctuated during the season, with little net trend in TMAC between mid-January and mid-February for these two cultivars. In nearly all cultivars, there was a small increase in anthocyanin content between the end of January and the middle of February. Viewed holistically, these results appear to show that the time of picking within the blueberry season may have a significant impact on the chemical composition of Australian-grown blueberries (in terms of both anthocyanin and antioxidant content); however, the specific impact of harvest time depends on the cultivar in question. Given that these compounds are largely responsible for the well-known health benefits of blueberries, this suggests that the potential health benefits associated with the consumption of these berries could also vary throughout the growing season.

\section{Conclusions}

In this pilot study, we profiled the changes in total monomeric anthocyanin content and total antioxidant capacity in four highbush blueberry cultivars during three maturation stages. While the TAC of most cultivars decreased with increasing ripeness, that of Blue Rose increased markedly. The TMAC increased sharply between the medium-ripe and fully ripe maturity stages in all cultivars. Throughout the harvest season, the TAC and TMAC of ripe fruit generally fluctuated over time, with the exact trends appearing to be cultivarspecific. This suggests that the levels of health-benefitting compounds in Australian-grown highbush blueberries will depend not only on the cultivar, but also upon the time of harvest. Although not explored in the present study, agronomic conditions are also likely to have a considerable impact on these compounds.

The spectrophotometric methods used for the measurement of TAC and TMAC in this study benefit from their speed and ease of use. This makes them suited to the rapid analysis of phytochemical contents in a large number of food samples, such as those included in this study. However, they are likely to be less specific compared to separation-based methods such as high-performance liquid chromatography (HPLC). Hence future work could focus on comparing the accuracy and precision of spectrophotometric and HPLC-based methods for the analysis of TMAC and specific antioxidant compounds (e.g., phenolic acids). Future studies could also investigate the temporal variation of TAC and TMAC over longer time periods than those included in the present study. 
Author Contributions: Conceptualization, L.W. and M.N.; methodology, M.S. and M.N.; software, J.B.J.; validation, M.S.; formal analysis, J.B.J.; investigation, M.S., S.R. and S.A.; resources, M.N.; data curation, S.A. and J.B.J.; writing-original draft preparation, J.B.J.; writing-review and editing, J.B.J., M.S., J.S.M., S.R., S.A., L.W. and M.N.; visualization, J.B.J.; supervision, L.W. and M.N.; project administration, L.W. and M.N.; funding acquisition, L.W. and M.N. All authors have read and agreed to the published version of the manuscript.

Funding: This research received no specific grant from any funding agency in the public, commercial, or not-for-profit sectors.

Institutional Review Board Statement: Not applicable.

Informed Consent Statement: Not applicable.

Data Availability Statement: The data presented in this study are available on request from the corresponding author.

Acknowledgments: We thank Buninyong Blueberry Farm (https://www.buninyongblueberries. com.au/) for providing the blueberry samples used in this study. The assistance of the Biomedical Sciences team at Federation University is also gratefully acknowledged.

Conflicts of Interest: The authors declare no conflict of interest.

\section{References}

1. Australian Blueberries. Blueberry Facts. Available online: https://australianblueberries.com.au/is-good/berry-facts/ (accessed on 24 December 2020).

2. Kalt, W.; Cassidy, A.; Howard, L.R.; Krikorian, R.; Stull, A.J.; Tremblay, F.; Zamora-Ros, R. Recent Research on the Health Benefits of Blueberries and Their Anthocyanins. Adv. Nutr. 2019, 11, 224-236. [CrossRef] [PubMed]

3. Rutledge, G.A.; Sandhu, A.K.; Miller, M.G.; Edirisinghe, I.; Burton-Freeman, B.B.; Shukitt-Hale, B. Blueberry phenolics are associated with cognitive enhancement in supplemented healthy older adults. Food Funct. 2021, 12, 107-118. [CrossRef] [PubMed]

4. Stevenson, D.; Scalzo, J. Anthocyanin composition and content of blueberries from around the world. J. Berry Res. 2012, 2, 179-189. [CrossRef]

5. Lohachoompol, V.; Mulholland, M.; Srzednicki, G.; Craske, J. Determination of anthocyanins in various cultivars of highbush and rabbiteye blueberries. Food Chem. 2008, 111, 249-254. [CrossRef]

6. Grace, M.H.; Xiong, J.; Esposito, D.; Ehlenfeldt, M.; Lila, M.A. Simultaneous LC-MS quantification of anthocyanins and nonanthocyanin phenolics from blueberries with widely divergent profiles and biological activities. Food Chem. 2019, 277, 336-346. [CrossRef] [PubMed]

7. Kalt, W.; Lawand, C.; Ryan, D.A.J.; McDonald, J.E.; Donner, H.; Forney, C.F. Oxygen Radical Absorbing Capacity, Anthocyanin and Phenolic Content of Highbush Blueberries (Vaccinium corymbosum L.) during Ripening and Storage. J. Am. Soc. Hortic. Sci. 2003, 128, 917. [CrossRef]

8. Castrejón, A.D.R.; Eichholz, I.; Rohn, S.; Kroh, L.W.; Huyskens-Keil, S. Phenolic profile and antioxidant activity of highbush blueberry (Vaccinium corymbosum L.) during fruit maturation and ripening. Food Chem. 2008, 109, 564-572. [CrossRef]

9. Spinardi, A.; Cola, G.; Gardana, C.S.; Mignani, I. Variation of Anthocyanin Content and Profile Throughout Fruit Development and Ripening of Highbush Blueberry Cultivars Grown at Two Different Altitudes. Front. Plant Sci. 2019, 10, 1045. [CrossRef] [PubMed]

10. Connor, A.M.; Luby, J.J.; Tong, C.B.S.; Finn, C.E.; Hancock, J.F. Genotypic and Environmental Variation in Antioxidant Activity, Total Phenolic Content, and Anthocyanin Content among Blueberry Cultivars. J. Am. Soc. Hortic. Sci. 2002, 127, 89. [CrossRef]

11. Routray, W.; Orsat, V. Blueberries and Their Anthocyanins: Factors Affecting Biosynthesis and Properties. Compr. Rev. Food Sci. Food Saf. 2011, 10, 303-320. [CrossRef]

12. Lohachoompol, V.; Srzednicki, G.; Craske, J. The change of total anthocyanins in blueberries and their antioxidant effect after drying and freezing. J. Biomed. Biotechnol. 2004, 2004, 248. [CrossRef] [PubMed]

13. Johnson, J.; Collins, T.; Walsh, K.; Naiker, M. Solvent extractions and spectrophotometric protocols for measuring the total anthocyanin, phenols and antioxidant content in plums. Chem. Pap. 2020, 74, 4481-4492. [CrossRef]

14. Die, J.V.; Jones, R.W.; Ogden, E.L.; Ehlenfeldt, M.K.; Rowland, L.J. Characterization and Analysis of Anthocyanin-Related Genes in Wild-Type Blueberry and the Pink-Fruited Mutant Cultivar 'Pink Lemonade': New Insights into Anthocyanin Biosynthesis. Agronomy 2020, 10, 1296. [CrossRef] 